\title{
Natural and Synthetic Glass and Crystal Reference Materials for Trace Element Microanalysis
}

\author{
William O. Nachlas ${ }^{1}$ \\ ${ }^{1 .}$ Department of Earth Sciences, Syracuse University, Syracuse, NY, USA 13244
}

Several new petrogenetic techniques have been developed in recent years that use the solubilities and diffusivities of trace elements in minerals to estimate the temperatures, pressures, and timescales of crystal formation. These single mineral thermometers, barometers, and geospeedometers rely on the concentration and distribution of trace elements and have been widely used in the geologic community because of the relative ease with which they can be applied; analysis of one component in a mineral phase can yield a wealth of information on geologic formation conditions. However, these techniques require measurements of ppm-level variations from $\mu \mathrm{m}$-scale regions of solid samples, and there are numerous analytical challenges that can arise when making measurements at these scales. To evaluate confidence in measurements at the limits of analytical resolution, it is essential to incorporate reference materials into the analysis routine to determine the accuracy and precision of trace element measurements.

This presentation evaluates experimentally synthesized and naturally occurring materials for use as trace element reference materials, with particular emphasis on trace element analysis of quartz. Quartz is one of the most commonly used phases for trace element petrogenetic techniques, and its simple mineralogy enables it to be utilized as a reference material for many other silicate phases. Analyses of synthetic quartz crystals and silica glasses are compared with natural quartz collected from field localities where it has been found to contain uniform trace element contents. The suitability of these materials as reference materials depends on the analysis methods employed. In some methods, it is essential to have a matrixmatched reference material, whereas others are insensitive to matrix effects. Some methods require multiple reference materials with compositions that span the range of compositions expected in unknown samples, whereas other methods can be evaluated with a single material of known composition. In some cases, it is useful to test analytical confidence using a "blank" (e.g. [1]), a reference material that is matrix-matched with the unknown sample but is devoid of the analyte of interest. In order to produce trace element reference materials that are widely-available to the analytical community and suitable for a variety of different measurement methods, this presentation discusses experimental and natural samples characterized by a variety of in-situ (EPMA, SIMS, LA-ICP-MS, SEM-CL, FTIR) and bulk (ICP-OES, XRD) measurement techniques.

Synthetic crystal reference materials were grown under controlled pressure-temperature conditions using a piston-cylinder device. This method produces crystals with trace element concentrations predicted for the solubility at the experimental conditions, and by conducting experiments over a range in pressuretemperature space, it is possible to prepare materials with compositions similar to those expected for many geologic environments. This method was used to produce synthetic quartz crystals with $\mathrm{Ti}$ and $\mathrm{Al}$ concentrations ranging from $\sim 10-1000 \mu \mathrm{g} \mathrm{g} \mathrm{g}^{-1}$. Each experiment produces $\sim 10^{3}$ individual crystals that range in size from 100-1000 $\mu \mathrm{m}$. EPMA of intragrain Ti concentrations in synthetic quartz crystals reveals uniform compositions within grain interiors (Figure 1). Because piston-cylinder experiments are conducted with high-purity starting materials, it is possible to control the composition of synthetic crystals within the limits of experimental conditions. 
A complementary approach for preparing microanalytical reference materials is to fabricate silica glasses doped with precise quantities of the analyte of interest. An experimental procedure has been developed that utilizes the high retentivity of nanoporous silica gel as a doping substrate [2]. Silica gel contains a network of nanopores that exert a capillary force on polar dopant molecules, as well as surface silanol groups that have a strong affinity for the dopant species. This method is capable of preparing populations of silica gel particles doped at a concentration that is within $10 \mu \mathrm{g} \mathrm{g}^{-1}$ of a target value. Doping solutions are obtained as plasma standard solutions that can be acquired commercially in a range of concentrations for many different elements. The use of silica gel as a doping substrate enables synthesis of a glass containing a selected element at a specified concentration and presents a method for preparing reference materials with precise compositional control.

Natural quartz crystals from select localities have been tested as reference materials for trace element analysis. Previous studies have characterized natural quartz and found consistent concentrations of trace elements and proposed their use as reference materials $[3,4]$. Hydrothermal quartz present in vugs in dolostone from Herkimer County, NY is useful as a trace element blank because it contains very low concentrations of most trace elements. SIMS analysis of $\mathrm{Ti}$ contents in Herkimer quartz indicate concentrations of 4-5 $\pm 2 \mathrm{ng} \mathrm{g}^{-1}$ [5], and EPMA of Ti and $\mathrm{Al}$ contents in Herkimer quartz crystals ranging in size from $50 \mu \mathrm{m}$ to $5 \mathrm{~cm}$ yields concentrations below detection limits for all samples. The abundance and wide availability of Herkimer quartz and its consistently-low trace element contents qualify it as an ideal blank reference material for trace element analysis.

\section{References:}

[1] JJ Donovan, HA Lowers and BG Rusk, American Mineralogist 96 (2011), p. 274-282.

[2] WO Nachlas, Geostandards and Geoanalytical Research 40 (2016), p. 505-516.

[3] A Audétat et al, Geostandards and Geoanalytical Research 39 (2014), p. 171-184.

[4] AM Cruz-Uribe et al, Geostandards and Geoanalytical Research (in press).

[5] S Kidder, J-P Avouac and Y-C Chan, Solid Earth 4, p. 1-21.
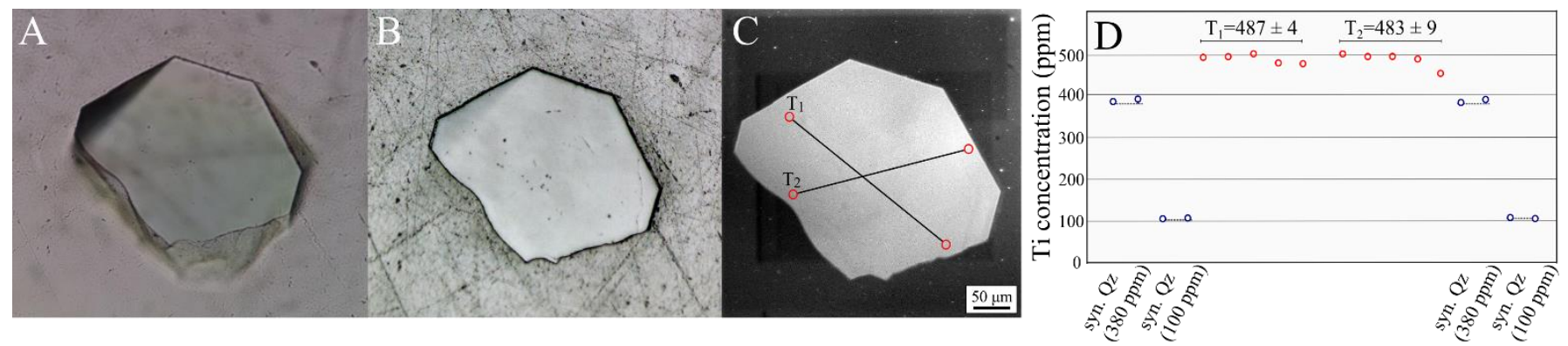

Figure 1. Analysis of a synthetic quartz crystal from a piston-cylinder experiment. Transmitted light (A) and reflected light (B) micrographs show a quartz single crystal mounted in epoxy and polished. A cathodoluminescence image (C) reveals a relatively uniform intragrain composition, which is supported by EPMA results (D) of two analytical transects that reveal consistent Ti concentrations. Accuracy and precision of Ti measurements are evaluated based on repeat measurements of independently-measured synthetic quartz reference materials (containing 380 and 100 ppm Ti). 